\title{
Investigations on Crystallisation Processes of Three Oxidic Gasifier Slag Systems
}

\author{
Jan Peter Schupsky ${ }^{1}$, Muxing Guo ${ }^{2}$, Bart Blanpain ${ }^{2}$, Michael Müller ${ }^{1}$ \\ ${ }^{1}$ Institute of Energy- and Climate Research - IEK 2, Forschungszentrum Jülich GmbH, Wilhelm-Johnen-Straße, 52428 \\ Jülich, Germany, j.schupsky@fz-juelich.de, mic.mueller@fz-juelich.de \\ ${ }^{2}$ Department of Materials Engineering, KU Leuven, 3001 Leuven, Belgium, muxing.guo@ @uleuven.be, \\ bart.blanpain@kuleuven.be
}

Technical Topics: Fluid Particle Fundamentals; Combustion and Gasification

\begin{abstract}
In entrained flow gasifiers, the production of oxidic slag accompanies the gasification process. This slag forms a layer on the refractory walls, flows downwards gravitationally and is collected in a water quench. Hence, the slag flow must be constant, since a slag blockage represents a worst-case-scenario. Crystallisation of the slag increases slag viscosity, leading to a possible slag blockage subsequently. Therefore, crystallisation processes in oxidic slags need to be understood and hence investigated.

In this study, three artificial, coal ash related oxidic slag systems were analysed on their crystallisation behaviour. Therefore, their melt behaviour was investigated via hot stage microscopy and differential thermal analysis (DTA). Additional thermochemical calculations were performed to predict crystallised phases. Subsequently, quenching experiments were conducted to generate supercooled crystallisation in the slag samples. These samples were analysed afterwards via XRD and SEM and the morphologies of crystals were characterized/described. In-situ observations on crystallisation growth were performed by using a confocal laser scanning microscope (CLSM). Finally, crystallised phases were compared with results obtained from thermochemical calculations and the impact of kinetics on the distributed phases was discussed. The knowledge on the crystallisation behaviour of various phases can be transferred to other slag systems, and can improve general crystallisation predictions made by thermochemical calculations.
\end{abstract}

\section{Introduction}

History of mankind is strongly influenced by the availability, quality and usage of energy sources. Burning of wood and charcoal allowed human beings to generate heat for cooking food and smelting copper for primitive weapons or agricultural tools [1]. However, in the recent centuries, the variation of fuels used and the applied technologies changed industrialization, economics and the environment subsequently. Meanwhile, there is wide spread use of lignite, coal, oil, gas as well as biomass in our modern technological world. Fuel gasification represents a suitable technology for energy production. Gasifiers have the advantage of flexible load conditions and relatively fast start up and shut down times. As a result, energy production from gasification can fill fluctuation gaps in power grids [2]. Especially the disadvantages of renewable energies, i.e. weather based fluctuations, can be strongly supported by gasification technology.

In slagging gasifiers, the melting of fuel ash represents a crucial parameter during operation. Due to high gasification temperatures up to $1600{ }^{\circ} \mathrm{C}$, fuel ash is present in a liquid state, the so called slag [3-5]. The slag forms a layer on top of the refractory materials and protects it from corrosion. However, a constant slag tapping is required to prevent a slag blocking in the gasifier [6]. As long as slag viscosity lies in a low viscous range below $25 \mathrm{~Pa}^{*}$ s, a constant slag flow with subsequent slag quenching can be ensured [7]. Thus, a viscosity increase in the slag obstructs the flow and the slag could solidify on the refractory walls, leading to a shut down. To predict the slag properties (e.g. viscosity), several models have been developed [8-10]. Although viscosity of fully liquid slag systems can be calculated sufficiently, its calculation for partly crystalline slag systems are of greater challenge [6,11]. Recent studies [12-15] indicated the impact of crystallisation on the slags viscosity.

In this study, crystallisation processes in three synthetic slag systems were investigated. The slags melting and crystallisation behaviours were investigated by hot stage microscopy and differential thermal analysis (DTA). Crystallisation products were ascertained by performing equilibrium calculations using the software package FactSage and the GTox database. As real processes are influenced by kinetics, experimental investigations were carried out to 
validate the impact of kinetics on crystallisation. Therefore, a quenching experiment was performed. Slag samples were supercooled, hold isothermally and quenched to specify the crystal content. Kinetic information was plotted in TTT (temperature-time-transformation) diagrams and the crystallised phases were analysed using XRD and SEMEDX. One of the synthetic slag system was additionally examined using confocal laser scanning microscopy (CLSM) [16]. CLSM delivers an in-situ view of crystallisation processes and documents crystal growth and morphology highly detailed. Finally, a comparison of the crystallised slags with FactSage calculations were made, to identify the impact of kinetics on the crystallisation.

\section{Experimental}

The investigated slag systems are based on coal ash analysis from previous work [12, 17]. Three systems were selected, based on their composition, acidity, viscosity and probable crystallised phases. To prevent compound volatility and to ensure a homogeneous bulk, compositions were set to $\mathrm{SiO}_{2}-\mathrm{Al}_{2} \mathrm{O}_{3}-\mathrm{CaO}-\mathrm{Fe}_{2} \mathrm{O}_{3}-\mathrm{MgO}$ systems (Tab. 1). ST-D-2 and HKT slags represent high acidic systems, with higher viscosities due to the presence of the network formers $\mathrm{SiO}_{2}$ and $\mathrm{Al}_{2} \mathrm{O}_{3}$. In contrast, HKR slag system is a basic system with high contents of $\mathrm{CaO}, \mathrm{Fe}_{2} \mathrm{O}_{3}$ and $\mathrm{MgO}$ and low viscosity.

Table 1: Slag composition of the three investigated synthetic slag systems based on ICP-OES analysis.

\begin{tabular}{|l|c|c|c|c|c|}
\hline Wt-\% & $\mathbf{S i O}_{\mathbf{2}}$ & $\mathbf{A l}_{\mathbf{2}} \mathbf{O}_{\mathbf{3}}$ & $\mathbf{C a O}$ & $\mathbf{F e}_{2} \mathbf{O}_{3}$ & MgO \\
\hline ST-D-2 & 60,9 & 21,6 & 10,6 & 6,9 & \\
\hline HKT & 54,7 & 22,3 & 14,6 & 2,6 & 5,8 \\
\hline HKR & 34,8 & 12,0 & 27,4 & 14,5 & 11,4 \\
\hline
\end{tabular}

Thermochemical calculations were performed by using Equilib module of the software package FactSage in combination with the version 7.2. GTox database [18], equilibrium calculations were performed to predict possible crystallised phases. As a boundary condition, the partial pressure of oxygen was set be in the reducing regime $\mathrm{p}\left(\mathrm{O}_{2}\right)=$ $10^{-12}$ bar.

Hot stage microscopy was carried out in a horizontal furnace (Rubotherm EB04/30, Bochum, Germany), based on guideline ASTM D 1857. Slag powders were pressed in mm-sized cylindrical pellets. The pellets were observed using a CCD camera and a customised programme took frequent pictures of the sample. Melting procedure during sample heating leads to several typical morphology associated temperatures: deformation (DT), softening (ST), hemispherical (HT) and flow (FT) [19].

Melting and crystallisation behaviour was additionally measured using differential thermal analysis (DTA). As the samples experience heating and cooling slopes, the exothermal behaviour is detected. Phase transformation (melting, crystallisation/ solidification) results in enthalpy changes, leading to peak formation in the DTA signal. A STA 449 F3 Jupiter (Netzsch, Selb, Germany) and $\mathrm{Al}_{2} \mathrm{O}_{3}$ crucibles were used, heating and cooling rates of $10 \mathrm{~K} / \mathrm{min}$ as well as an $\mathrm{Ar}, \mathrm{Ar} / 4 \% \mathrm{H}_{2}$ atmosphere was applied to ensure reducing gasifier conditions, respectively.

Beforehand the quenching experiment, the slag samples were annealed at $1550{ }^{\circ} \mathrm{C}$ for 5 hours in Mo-crucibles, to ensure sample homogeneity. The quenching experiment was conducted in a vertical high temperature furnace (HTF 18/8, Carbolite Gero GmbH \& Co. KG, Neuhausen, Germany). Under reducing Ar/4\% $\mathrm{H}_{2}$ atmosphere, the samples experienced heating to re-melt the slag, followed by supercooling below the liquidus temperature. The cooling slopes were set as $-7 \mathrm{~K} / \mathrm{min}$ and isothermal steps with a resolution of $50{ }^{\circ} \mathrm{C}$ were chosen. Samples were quenched with time dependency. Depending on incubation time, time steps were set either in double digit minute scales or in hour scales (maximum $24 \mathrm{~h}$ ) to finally quench the samples into water.

Post-experimental analysis was conducted by microscopy, XRD and SEM-EDX analysis. To describe the slags inner structure, a KEYENCE VHX-S550E digital microscope (KEYENCE DEUTSCHLAND GmbH, Neu-Isenburg, Germany) was used. Amorphous solidified slag samples were distinguished from crystallised ones and high resolution images were taken. Based on these separations, crystallised samples were further analysed. XRD measurements were applied using a Panalytical EXMPYREAN (Malvern Panalytical, Almelo, Netherlands) device on representative samples. Spectra peaks were correlated using datasets from ICSD (Inorganic Crystal Strucre Databse, FIZ Karlsruhe, Germany) database. The morphology and chemistry of crystallised phases were determined by SEM-EDX analysis, using Zeiss Merlin II and Supra 50 VP (Carl Zeiss Microscopy GmbH, Jena, Germany) devices. High-Res images, linescans and element-mappings were used to identify the crystallised phases.

Additionally, a CLSM experiment (Materiaalkunde dep., KU Leuven, Belgium) was conducted to determine crystallisation kinetics and crystal growth in further detail. CLSM allows in-situ observations of samples with 
magnifications of up to $x 200$. The set up basically consists of a high temperature furnace chamber, heated by a halogen lamp, an optical unit, including a CCD camera and a class $2 \mathrm{He}-\mathrm{Ne}$ laser and finally computational and digital control units for operation. A selection of samples was further investigated by SEM-EDX afterwards.

\section{Results}

Initial investigations on the melt behaviour were conducted by hot stage microscopy and are shown in Table 2. As determined by the HT, significant melting of the slag samples occurred below $1376^{\circ} \mathrm{C}$. HKR slag samples displayed the lowest HT of $1301{ }^{\circ} \mathrm{C}$, while ST-D-2 instead showed the highest HT $\left(1376^{\circ} \mathrm{C}\right)$. FT exceeded HT slightly and was determined at $1316{ }^{\circ} \mathrm{C}, 1340{ }^{\circ} \mathrm{C}$ and $1414{ }^{\circ} \mathrm{C}$, respectively. These findings allow the definition of a necessary peak temperature the samples have to experience to ensure complete melting during pre-treatment. The peak temperatures of sample annealing (pre-treatment) was therefore set to $1550{ }^{\circ} \mathrm{C}$, to guarantee homogeneous melting.

Table 2: Hemispherical (HT) and flow temperature (FT) of oxidic slag samples

\begin{tabular}{|l|c|c|}
\hline & $\begin{array}{c}\text { Hemispherical Temperature } \\
(\mathbf{H T})\end{array}$ & Flow temperature (FT) \\
\hline ST-D-2 & $1376^{\circ} \mathrm{C}$ & $1414^{\circ} \mathrm{C}$ \\
\hline HKT & $1314^{\circ} \mathrm{C}$ & $1340^{\circ} \mathrm{C}$ \\
\hline HKR & $1301{ }^{\circ} \mathrm{C}$ & $1316^{\circ} \mathrm{C}$ \\
\hline
\end{tabular}

Sample annealing led to the formation of a homogeneous bulk that is supposed to solidify amorphous. To verify the melting properties of this solidified slag bulk, additional DTA measurements were conducted. In this case, HKT slag was chosen as an example to describe general findings. The slags were investigated by applying several loop measurements subsequently after each other. After initial heating, slag is expected to form a homogeneous bulk which melted again in the second loop and so forth. As indicated in Fig. 1, melting of homogeneous HKT slag occurs between approx. $1110^{\circ} \mathrm{C}$ and $1265^{\circ} \mathrm{C}$. The shape of DTA signal displays asymmetric peak shape, indicating that several phases were present in the slag. Therefore, the previous cooling slope may lead to the formation of crystallised phases, which melt at different temperatures. ST-D-2 slag sample indicated melting between $1215^{\circ} \mathrm{C}$ and $1285^{\circ} \mathrm{C}$. HKR showed slag creeping inside the crucible and strong reactions with the crucible material. As a result DTA signals were not reliable and not used in this study. Compared to hot stage microscopy results of slag powder, the melting point of homogeneous slag is lower. Furthermore, hot stage microscopy and DTA prove that $1450{ }^{\circ} \mathrm{C}$ as the peak temperature of the quenching furnace are sufficient to create a homogeneous liquid slag state.

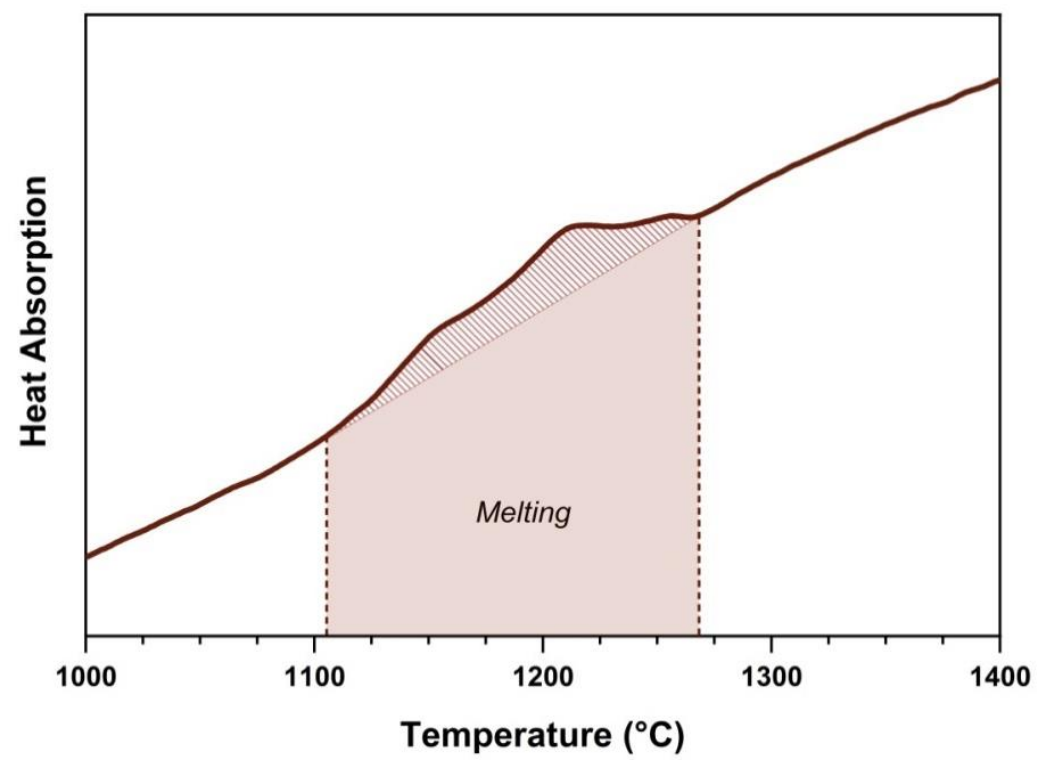

Figure 1: DTA signal of homogeneous HKT heating profile. 
To predict crystallised phases in the slag systems, thermodynamic equilibrium calculations were executed (Fig. 2). To display reducing conditions, oxygen partial pressures were set to $10^{-12}$ bar. Due these reducing conditions, Equilib predicts the formation of reduced iron in the slag. Accordingly, two slag phases were calculated, especially in the HKR system. ST-D-2 slag system shows initial crystallisation at approx. $1315{ }^{\circ} \mathrm{C}$ and crystallises completely at $1005{ }^{\circ} \mathrm{C}$. Anorthite $\left(\mathrm{CaAl}_{2} \mathrm{Si}_{2} \mathrm{O}_{8}\right)$ crystallises at the highest temperature, followed by $\mathrm{SiO}_{2}$, which crystallised in cristobalite structure. Cordierite is the final phase that should grow. Anorthite fraction reaches roughly $50 \%$ as the slag is fully solid. $\mathrm{SiO}_{2}$ is the second most abundant phase in that system. HKT slag system indicates comparable crystallisation products, compared to ST-D-2 (Fig. 2). Anorthite is the dominant crystalline phase, followed by cordierite and $\mathrm{SiO}_{2}$. At lower temperatures of approx. $1130^{\circ} \mathrm{C}$ clinopyroxene initially crystallises. The overall solidification of liquid slag ends at $1060{ }^{\circ} \mathrm{C}$. As ST-D-2 and HKT have comparable compositions (except for the presence of MgO), it can be argued that the coincidence of the crystallisation products is not surprising. However, equilibrium calculations predict dissimilar crystallised phases for the HKR slag system. As Fig. 2 indicates, melilite firstly crystallises at temperatures of $1330^{\circ} \mathrm{C}$. With a decline in temperature, melilite content in the slag fluctuates until solidification is reached at 1125 ${ }^{\circ} \mathrm{C}$. Regarding to Equilib predictions, olivine is the dominant phase reaching a fraction of $60 \%$ at the solidus temperature. At an intermediate temperature range between $1150{ }^{\circ} \mathrm{C}$ and $1240{ }^{\circ} \mathrm{C}$, small fractions of spinel are supposed to crystallise. At very low temperatures, anorthite is expected to crystallise as well.

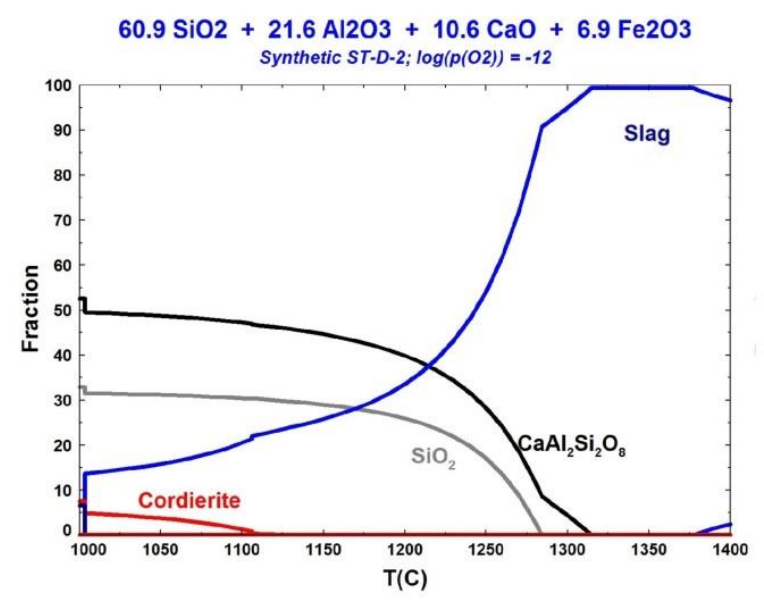

$54.7 \mathrm{SiO} 2+22.3 \mathrm{Al} 2 \mathrm{O} 3+14.6 \mathrm{CaO}+2.6 \mathrm{Fe} 2 \mathrm{O} 3+5.8 \mathrm{MgO}$
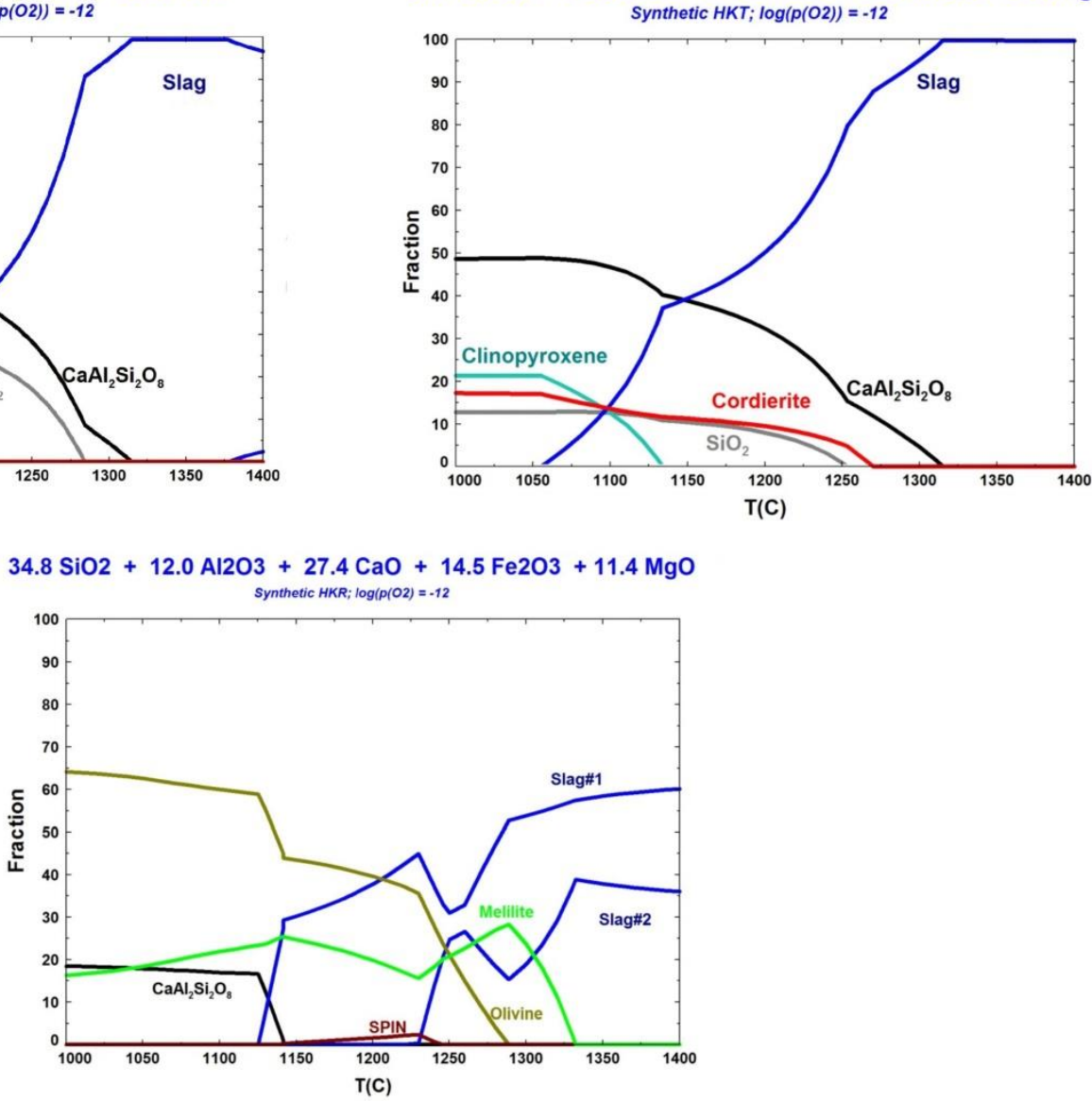

Figure 2: FactSage Equilib calculations of synthetic ST-D-2, HKT and HKR slag systems. Calculations were executed using the GTox database and applying $p(O 2)=10^{-12}$ bar.

According to the slags melting and crystallisation characteristics mentioned above, the experimental validation with kinetic data is the subsequent step. Therefore, quenching experiments were performed with all slag systems. The time intervals chosen were $0 \mathrm{~h}, 1 \mathrm{~h}, 4 \mathrm{~h}, 7 \mathrm{~h}$ and $24 \mathrm{~h}$. Though, ST-D-2 and HKT could be analysed quite well with these parameters, HKR slag showed rapid incubation and crystallisation. As a result, time steps were reduced to 10 min and $30 \mathrm{~min}$ for this system (Fig. 3). After the quenching experiment, samples were cut into hemispheres, embedded into epoxy resin, grinded and polished. Ultimately, samples were examined by microscopy to identify crystalline phases. According to this information, TTT diagrams were plotted for the slag systems. As kinetic data of ST-D-2 and HKT 
indicate comparable kinetic data, only HKT and HKR are displayed in Fig. 3. The comparison of both slag systems shows different tendencies. HKT (and ST-D-2) indicates slow incubation time of $1 \mathrm{~h}$ (at $1150{ }^{\circ} \mathrm{C}$ ). However, in this early state only small regions were influenced by crystallisation. In general, the overall sample of HKT and ST-D-2 showed great spatial distributed crystallisation after $4 \mathrm{~h}$ and $7 \mathrm{~h}$ of holding time. A comparison with equilibrium calculations (Fig. 2) indicates that the solidus temperatures of the slag systems were strongly shifted to lower temperatures of $900{ }^{\circ} \mathrm{C}$. This solidus retardation clarifies the impact of kinetics on the solidification of supercooled liquids. In contrast to HKT, HKR slag showed immediate crystallisation after $1200{ }^{\circ} \mathrm{C}$. Therefore, holding times were reduced to several minutes. Crystallisation kinetics were significantly higher. As a result, crystallisation was widespread at the very first samples quenched at $1150{ }^{\circ} \mathrm{C}$ and $1100{ }^{\circ} \mathrm{C}$. In the vertical high-temperature furnace, achievable cooling rates were not high enough to supercool HKR slag before the isothermal steps were reached. Therefore, CLSM experiment was chosen to observe crystallisation in HKR slag.
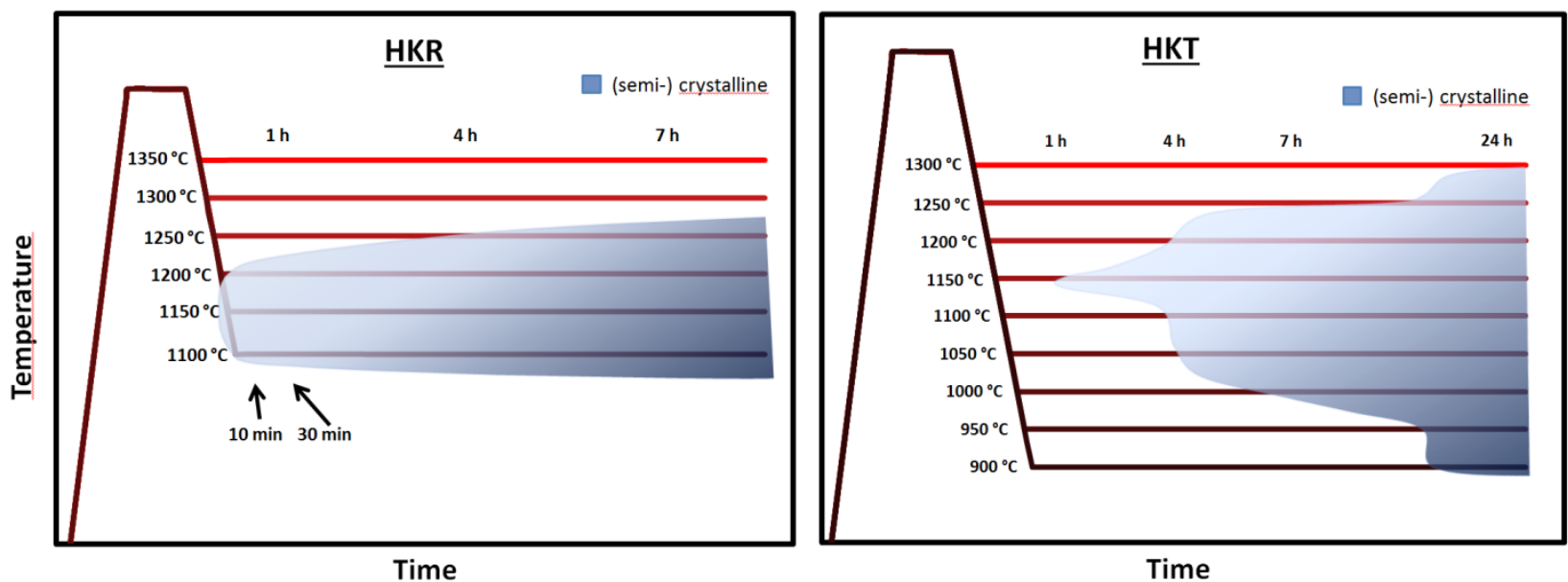

Figure 3: TTT diagrams of synthetic HKR and HKT slag. Crystalline "nose" is displayed by blueish area. Cooling slope was chosen as $-7 \mathrm{~K} / \mathrm{min}$.

By analysing HKR slag with CLSM, incubation times could be determined sufficiently. In pre-experiments, even high double digit cooling slopes led to spontaneous crystallisation in the slag. Thus, maximum cooling rates of $-250 \mathrm{~K} / \mathrm{min}$ were applied in the experiment to ensure sufficient cooling of HKR samples before the isothermal segment started. As Table 3 indicates, incubation time of HKR slag is very low. While measurements at $1250{ }^{\circ} \mathrm{C}$ indicate no crystallisation after almost three hours of holding time, a declined temperature of $50{ }^{\circ} \mathrm{C}$ to $1200{ }^{\circ} \mathrm{C}$ led to initial crystallisation after $270 \mathrm{~s}$. Nonetheless, this temperature allows an observation of crystallisation over a time period of several double digit minutes. As a temperature of $1100{ }^{\circ} \mathrm{C}$ was reached, the slag crystallised fully after $120 \mathrm{~s}$ of holding time. After the crystal incubation time, the time period passed until a crystal arrangement is formed was very minuscule. These findings prove that the understanding of crystallisation in oxidic slags is a crucial parameter for the stability of gasification processes $[6,12,13]$.

Table 3: CLSM data about incubation time and crystallisation of HKR slag. Crystallisation grade was estimated from CLSM images.

\begin{tabular}{|l|c|c|c|c|}
\hline & $1250{ }^{\circ} \mathrm{C}$ & $1200{ }^{\circ} \mathrm{C}$ & $1150{ }^{\circ} \mathrm{C}$ & $110{ }^{\circ} \mathrm{C}$ \\
\hline Incubation time & $>170 \mathrm{~min}$ & $270 \mathrm{~s}$ & $180 \mathrm{~s}$ & $30 \mathrm{~s}$ \\
\hline $\begin{array}{l}\text { Interconnected crystal arrangement } \\
\text { in liquid slag }\end{array}$ & unknown & $65 \mathrm{~min}$ & $21 \mathrm{~min}$ & $40 \mathrm{~s}$ \\
\hline Slag fully crystallised & unknown & unknown & unknown & $120 \mathrm{~s}$ \\
\hline
\end{tabular}

Ultimately, the crystallised phases were investigated using XRD and SEM. Regarding to the higher meaningfulness of SEM-EDX results compared with XRD spectra, this study will expound on SEM-EDX data in the following. Three samples that experienced the same parameters (temperature and time) were chosen to enable a suitable comparison of its crystallisation content and its crystallisation processes. As seen in Fig. 4, crystallisation products display different appearances in the slags. ST-D-2 slag crystallises in a cloudish pattern with pale green colour. HKT slag crystallises as larger crystals with visible vitreous glassy slag in between the crystals. HKR slag displays a blackish colour with 
large scale crystals. The differences in the crystal sizes probably result in the different slags viscosities. Due to the significant lower content of network formers (lower acidity), viscosity is expected to be lower as well. Low viscosity entails high diffusion and therefore allows crystals to grow larger in the liquid slag.

As indicated by SEM images, crystallised phases in ST-D-2 slag show grainsizes of less than $25 \mathrm{~mm}$. The slag is made up of an elongated phase (greyish) and a roundish phase (blackish). SEM-EDX measurements (\#1 ST-D-2, Fig. 4) indicated that the roundish phase correlates with $\mathrm{SiO}_{2}$ that was predicted via FactSage as cristobalite. The elongated particles (\#2 ST-D-2) display very good agreement with anorthite composition. This phase was the most dominant phase in the collection of all ST-D-2 samples, also displaying an elongated shape, but with variations of its crystal sizes. The slag composition of remaining amorphous crystallised slag shows depletion of Ca due to the presence of anorthite phase and enrichment of Fe. As no Fe-containing phase was observed in this sample, this deviation of the slag composition can be explained.

HKT slag system crystallised alike ST-D-2 slag, though the macroscopic comparison seems to indicate differences. Anorthite (\#1 HKT, Fig.4) is the most abundant phase in all HKT slag samples. In the displayed sample of Fig. 4, clinoenstantite, a pyroxene phase was determined via SEM-EDX analysis. Other samples also indicate augite and pigeonite as variations of the pyroxene phase. This phase captures $\mathrm{Mg}$ - and $\mathrm{Fe}$ - contents, which are not incorporated in anorthite crystals. $\mathrm{A} \mathrm{SiO}_{2}$ phase was also determined, though not displayed in Fig. 4. While the pyroxene phase aims

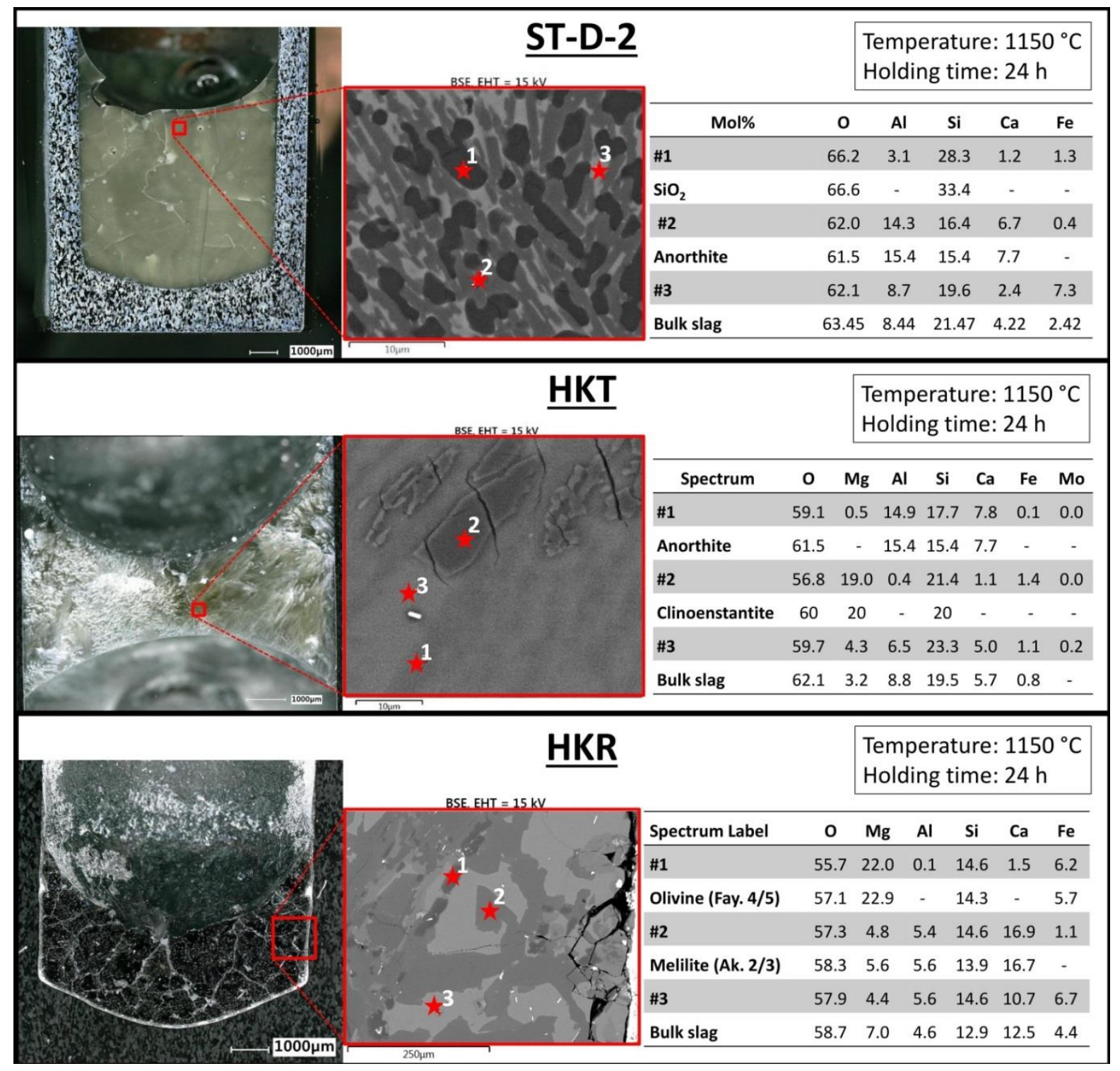

Figure 4: Comparison of three samples quenched at the same conditions. Investigations made by microscopy (left) and SEM-EDX measurements of crystallised phases. 
to form polygonal shapes in the intercrystalline gaps of anorthite. Anorthite displays elongated shapes as described before in ST-D-2, again. Repeatedly, $\mathrm{SiO}_{2}$ also crystallises in roundish particles.

According to different composition, acidity and viscosity of HKR slag, its crystallisation products are of dissimilar character (Fig. 4). The most present phase was determined as melilite. Due to its miscibility, melilite crystals show a varying composition between akermanite and gehlenite. This phase crystallised with the highest kinetic behaviour and forms large crystals of up to several mm length. In the cross section they appear as dendrites. However, microscopic and SEM-EDX analysis of CLSM samples of HKR slag determined a three dimensional, tetragonal pyramidal shape of the melilite phase. However, these pyramidal morphologies appear as rectangular dendrites in a cross section. The morphology of the detected olivine phase (\#1 HKR, Fig. 4) could only be determined by the analysis of cross sections. Olivine is mainly build-up of fayalite and forms polygonal shaped single crystals, which sometimes arrange in an elongated macroscopic shape. Spinel was also determined as a third phase (not displayed in Fig. 4). It shows clear prismatic shapes, but has the smallest crystal size of less than $50 \mathrm{~mm}$. Its overall content is also minor, compared to melilite and olivine.

As experiments indicate the kinetic influence on crystallisation in supercooled slags, a comparison with the thermodynamic calculations (Fig. 2) reveals similarities and differences between both approaches. In general, kinetic influence shifted the initial crystallisation temperature to lower temperatures in all slag samples. ST-D-2 and HKT samples also indicate that slag could be supercooled below the solidus temperature without any crystallisation occurring, due to their high viscosity. In detail, ST-D-2 slag shows the same crystallisation products (anorthite $+\mathrm{SiO}_{2}$ ) as predicted via FactSage. Also the dominance of anorthite was observed in the quenched sample. However, no cordierite phase was found in the slag (Fig. 2, 4). Instead, hercynite a Fe-rich spinel was found in one quenched sample, incorporating the enriched $\mathrm{Fe}$ in the remaining liquid slag. Nonetheless, phase prediction via FactSage Equilib calculations was satisfactory. As stated by Fig. 2, HKT slag should form comparable phases in the supercooled territory, complemented by a clinopyroxene phase. With the exception of cordierite, all phases were determined in the quenched samples. The clear dominance of anorthite was also observed. Thus, $\mathrm{SiO}_{2}$ and clinopyroxene crystallised mostly simultaneous at the same temperature conditions. Also in the case of HKT, thermodynamic predictions delivered satisfactory results, as long as the cordierite phase is neglected.

Also for HKR slag system, FactSage prediction based on GTox database delivered comparable results with kinetically influenced experiments. Melilite is the dominant phase above olivine and spinel in the quenching samples, though calculations stated olivine as the most dominant phase below $1250{ }^{\circ} \mathrm{C}$ (Fig. 2). The presence of anorthite was not determined in the analysed samples. The summary for HKR is similar compared to HKT and ST-D-2 - phase predictions via FactSage Equilib calculations and GTox database are satisfactory, as long as temperature shifts to lower temperatures and the exclude of the cordierite phase are taken into account.

\section{Conclusion}

In this study, crystallisation processes and crystallisation products of three synthetic gasifier slag systems were investigated and compared with thermodynamic calculations. The slag systems are characterised as two acidic systems (ST-D-2, HKT), containing high portions of $\mathrm{SiO}_{2}$ and $\mathrm{Al}_{2} \mathrm{O}_{3}$ and a system with higher basicity (HKR). All systems displayed crystallisation in the supercooled regime. However, kinetic retardation of incubation time is strongly varying between an hour-scale in the case of ST-D-2 and HKT and a minute/second-scale in the case of HKR. The impact of slag composition strongly influences incubation time and crystallisation tendencies. This finding is based on the slags diffusion, which is influenced by the slags viscosities, respectively. Low viscose HKR slag has a higher diffusion, allowing crystals to incorporate atoms faster from the liquid slag than higher viscose ST-D-2 and HKT systems.

Crystallisation products were determined via XRD and SEM-EDX analysis. Applying latter method, crystallised phases were intensively investigated. The ascertained phases were mostly predicted by FactSage equilibrium calculations using the GTox database. The major phases were present in the slag, as predicted by the equilibrium calculations, except cordierite. The lack of cordierite phase formation in the experiment could suffer from a kinetic barrier or its thermodynamic data used in the calculations are misleading. Anorthite and melilite are abundant phases in their systems, growing firstly and in large portions. Crystal size is also influenced by the slags viscosity, as lower viscosities support crystal growth due to higher atom mobility. 


\section{Acknowledgments}

This study has been performed in the framework of the HotVeGas Project supported by Bundesministerium für Wirtschaft und Technologie (FKZ 0327773).

\section{Literature}

[1] V. Smil, World history and energy, Encyclopedia of Energy, Elsevier, Amsterdam, 2004, pp. 549-561. [2] P.K. Johne, P.; Fendt, S.; Spliethoff, H. , Fuel \& Load-Flexible Entrained Flow Gasifier Operation \& Applications: Flame Parameters \& Flexible Burner 27th International Conference on the Impact of Fuel Quality on Power Production and the Environment, Lake Louise, Canada, 2018, pp. 13.

[3] S. Seebold, M. Eberhard, G. Wu, E. Yazhenskikh, D. Sergeev, T. Kolb, M. Müller, Thermophysical and chemical properties of bioliq slags, Fuel, 197 (2017) 596-604 DOI: https://doi.org/10.1016/j.fuel.2017.02.027.

[4] J.E.G.P. J.Th.G.M. Eurling, Process Performance of the SCGP at Buggenum IGCC, Gasification Technologies Conference, San Francisco, California, 1999, pp. 21.

[5] S. Vargas, F.J. Frandsen, K. Dam-Johansen, Rheological properties of high-temperature melts of coal ashes and other silicates, Progress in Energy and Combustion Science, 27 (2001) 237-429 DOI: https://doi.org/10.1016/S0360-1285(00)00023-X.

[6] S. Seebold, The influence of crystallization on the flow of coal ash-slags, FUEL, 187 (2017) 376-387 DOI: http://dx.doi.org/10.1016/j.fuel.2016.09.078.

[7] G. Wu, E. Yazhenskikh, K. Hack, E. Wosch, M. Müller, Viscosity model for oxide melts relevant to fuel slags. Part 1: Pure oxides and binary systems in the system SiO2-Al2O3-CaO-MgO-Na2O-K2O, Fuel Process. Technol., 137 (2015) 93-103 DOI: http://dx.doi.org/10.1016/j.fuproc.2015.03.025.

[8] A.F. Stam, W.R. Livingston, G. Brem, K.J.M. Cremers, Review of Models and Tools for Slagging and Fouling Prediction for Biomass Co-combustion, IEA Bioenergy Conference, 2010, pp. 12.

[9] A. Kondratiev, E. Jak, Review of experimental data and modeling of the viscosities of fully liquid slags in the Al2O3-CaO-'FeO'-SiO2 system, Metallurgical and Materials Transactions B, 32 (2001) 1015-1025 DOI: dx.doi.org/10.1007/s11663-001-0090-y.

[10] G. Wu, S. Seebold, E. Yazhenskikh, J. Tanner, K. Hack, M. Müller, Slag mobility in entrained flow gasifiers optimized using a new reliable viscosity model of iron oxide-containing multicomponent melts, Applied Energy, 236 (2019) 837-849 DOI: https://doi.org/10.1016/j.apenergy.2018.11.100.

[11] L. Zhuangzhuang, Z. Ling, M. Annelies, B. Bart, G. Muxing, Non-Newtonian behavior of solid-bearing silicate melts: An experimental study, J. Non-Cryst. Solids, 493 (2018) 65-72 DOI: https://doi.org/10.1016/j.jnoncrysol.2018.04.042.

[12] S. Seebold, Über den Einfluss der Kristallisation auf das Fließverhalten oxidischer Schmelzen, Fakultät für Maschinenwesen, Rheinisch-Westfälische Technische Hochschule Aachen, Aachen, 2017, pp. 168.

[13] D.H. Schwitalla, A.M. Bronsch, M. Klinger, S. Guhl, B. Meyer, Analysis of solid phase formation and its impact on slag rheology, Fuel, 203 (2017) 932-941 DOI: dx.doi.org/10.1016/j.fuel.2017.04.092.

[14] L. Zhuangzhuang, C. Liugang, M. Guo, B. Blanpain, Effect of Crystallization on the Abrupt Viscosity Increase during the Slag Cooling Process, ISIJ Int., 58 (2018) 1972-1978 DOI: https://doi.org/10.2355/isijinternational.ISIJINT-2018-176.

[15] L. Zhuangzhuang, M. Annelies, B. Bart, G. Muxing, Rheological Transitions of the Solid-Bearing Slag During Cooling Process, Metall. Mater. Trans. B-Proc. Metall. Mater. Proc. Sci., 49 (2018) 2649-2657 DOI: doi.org/10.1007/s11663-018-1324-6.

[16] P.T. Jones, D. Desmet, M. Guo, D. Durinck, F. Verhaeghe, J. Van Dyck, J. Liu, B. Blanpain, P. Wollants, Using confocal scanning laser microscopy for the in situ study of high-temperature behaviour of complex ceramic materials, Journal of the European Ceramic Society, 27 (2007) 3497-3507 DOI: https://doi.org/10.1016/j.jeurceramsoc.2007.01.022. 
[17] T. Melchior, Untersuchungen zur Oberflächenspannung von Kohleschlacken unter Vergaserbedingungen, Fakultät für Maschinenwesen, Rheinisch-Westfälische Technische Hochschule, Aachen, 2010, pp. 270.

[18] T.J. K. Hack, M. Müller, E. Yazhenskikh, G. Wu, A novel thermodynamic database for slag systems and refractory materials, Proceedings of the 5th International Congress on the Science and Technology of Steelmaking, Dresden, Germany, 2012.

[19] M. Dohrn, Influence of Load Changes on the Deposit Behavior during Combustion of Five Different Hard Coals, Energy and fuels, 32 (2018) 3985-3994 DOI: https://doi.org/10.1021/acs.energyfuels.7b04020.

\section{Figure numbers and captions}

Figure 5: DTA signal of homogeneous HKT heating profile.

Figure 6: FactSage Equilib calculations of synthetic ST-D-2, HKT and HKR slag systems. Calculations were executed using the GTox database and applying $\mathrm{p}(\mathrm{O} 2)=10-12$ bar.

Figure 3: TTT diagrams of synthetic HKR and HKT slag. Crystalline "nose" is displayed by blueish area. Cooling slope was chosen as $-7 \mathrm{~K} / \mathrm{min}$.

Figure 4: Comparison of three samples quenched at the same conditions. Investigations made by microscopy (left) and SEM-EDX measurements of crystallised phases.

\section{Table numbers and captions}

Table 1: Slag composition of the three investigated synthetic slag systems based on ICP-OES analysis.

Table 2: Hemispherical (HT) and flow temperature (FT) of oxidic slag samples

Table 3: CLSM data about incubation time and crystallisation of HKR slag. Crystallisation grade was estimated from CLSM images. 